\title{
EXISTENCE OF SOLUTIONS FOR VOLTERRA INTEGRAL INCLUSIONS
}

\author{
TAO ZHU, CHAO SONG AND GANG LI \\ Communicated by Hermann Brunner
}

\begin{abstract}
In this paper, we use different methods to investigate continuous solutions of the nonlinear Volterra integral inclusions. The results obtained here improve and generalize many known results.
\end{abstract}

1. Introduction. In this paper, we study the problem of nonlinear Volterra integral inclusions

$$
x(t) \in h(t)+\int_{0}^{t} k(t, s) F(s, x(s)) d s, \quad t \in[0,1],
$$

where $F:[0,1] \times \Re \rightarrow \Re$ is a multi-valued function with nonempty compact values. Throughout this paper, the map $x \rightarrow F(t, x)$ is either upper semi-continuous or lower semi-continuous for almost every $t \in[0,1]$.

A series of research results has appeared concerning this kind of nonlinear Volterra integral inclusion (1.1) in recent years $[\mathbf{2 - 6}, \mathbf{8}, \mathbf{1 1 - 1 9}]$. For example, In [12] Kisielewicz studies the multi-valued differential equation when $F$ is a multi-valued mapping taking as its values nonempty compact, but not necessarily convex, subsets in a separable Banach space. In [3], Agarwal and O'Regan establish the existence of $C[0, T)$ and $L_{\mathrm{loc}}^{p}[0, T)$ solutions to the Volterra integral inclusions (1.1). In [13] O'Regan obtains that the differential inclusions has a solution $u \in W^{1,1}([0, T], X)$ in a separable Banach space. In [15] O'Regan studies the nonlinear Volterra integral inclusions when $F$ is a condensing

\footnotetext{
Keywords and phrases. Fixed point theorem, Volterra integral inclusions, upper semi-continuous, lower semi-continuous.

The research was supported by Scientific Research Foundation of Nanjing Institute of Technology (No. QKJA2011009) and Natural Science Foundation of Jiangsu Province (No. 13KJB110011).

Received by the editors on March 25, 2012, and in revised form on March 1, 2013.

DOI:10.1216/JIE-2013-25-4-587 Copyright (C)2013 Rocky Mountain Mathematics Consortium
} 
map. O'Regan and Precup [16] establish existence principles for the integral inclusions (1.1) when $F$ satisfies Mönch's condition. O'Regan [14] presents existence results for nonlinear Volterra integral inclusions (1.1) when the map $x \rightarrow F(t, x)$ is either upper semi-continuous or lower semi-continuous for almost every $t \in[0, T]$. In [5], Avgerinos discusses the nonlinear Volterra integral inclusions when $F$ is a multivalued mapping taking as its values nonempty, $w$-compact and convex. In [19] Papageorgiou gives the existence of continuous solutions of the nonlinear Volterra integral inclusions (1.1) when the map $x \rightarrow F(t, x)$ is upper semi-continuous from $X$ into $X_{w}$ (where $X_{w}$ denotes the Banach space $X$ endowed with the weak topology). In [17] Papageorgiou studies the nonlinear Volterra integral inclusions when $F(t, \cdot)$ is Hausdorff continuous for all $t \in I$. The purpose of this paper is to continue the study of these authors. We present the existence result of (1.1) when there exists a function $m(t)$ satisfied an inequality. And, for some special functions, we prove there must exist an $m(t)$ that satisfies the above inequality. Therefore, our results improve and generalize the corresponding results in $[\mathbf{5}, \mathbf{6}, \mathbf{1 3 - 1 5}, \mathbf{1 7 - 1 9}]$.

The paper will be divided into two main sections. Section 2 gathers some known results on multi-valued maps. We will use these results in Section 3 to investigate the existence of continuous solutions of the nonlinear Volterra integral inclusions (1.1).

2. Preliminaries. Let $E_{1}$ and $E_{2}$ be two Banach spaces, $X$ a nonempty closed subset of $E_{1}$ and $S$ a measurable space (respectively $S=I \times \Re$, where $I$ is a real interval and $A \subseteq S$ is L $\otimes$ B measurable if $A$ belongs to the $\sigma$-algebra generated by all sets of the form $N \times D$ where $N$ is Lebesgue measurable in $I$ and $D$ is Borel measurable in $\Re$ ). Let $G$ : $S \rightarrow E_{2}$ and $H: X \rightarrow E_{2}$ be two multi-functions with nonempty closed values. The function $G$ is measurable (respectively $\mathrm{L} \otimes \mathrm{B}$ measurable) if the set $\{t \in S: G(t) \bigcap B \neq \varnothing\}$ is measurable for any closed set $B$ in $E_{2}$. The function $H$ is lower semi-continuous (l.s.c.) (respectively, upper semi-continuous (u.s.c.)) if the set $\{x \in X: H(x) \cap B \neq \varnothing\}$ is open (respectively, closed) for any open (respectively, closed) set $B$ in $E_{2}$. If $H$ is lower semi-continuous and upper semi-continuous, then $H$ is continuous. $H$ is compact if $\overline{H(X)}=\overline{\bigcup_{x \in X} H(x)}$ is compact in $E_{2}$, and it is completely continuous if $\overline{H(\Omega)}$ is compact for all bounded sets $\Omega \subseteq X$. A subset $A$ of $L^{1}([0,1] ; \Re)$ is decomposable if, for all $u, v \in A$ 
and $N \subseteq[0,1]$ measurable, the function $u_{\chi N}+v_{\chi[0,1] / N} \in A$.

Let $F:[0,1] \times \Re \rightarrow \Re$ be a multi-valued function with nonempty compact values. Throughout this paper, our maps $h, F$ and the kernel $k$ will satisfy some of the following properties:

(1) $h \in C[0,1]$, and let $a=\|h\|=\max _{0 \leq t \leq 1}|h(t)|$.

(2) For each $t \in[0,1], k(t, s)$ is measurable on $[0, t]$ and $k(t)=$ ess $\sup |k(t, s)|, 0 \leq s \leq t$, is bounded on $[0,1]$, let $K=\sup _{0 \leq t \leq 1}|k(t)|$. The map $t \rightarrow k_{t}$ is continuous from $[0,1]$ to $L^{\infty}[0,1]$; here $k_{t}(s)=$ $k(t, s)$.

(3) $t \rightarrow F(t, x)$ is measurable for every $x \in \Re . x \rightarrow F(t, x)$ is upper semi-continuous for almost every $t \in[0,1]$.

(4) $(t, x) \rightarrow F(t, x)$ is $\mathrm{L} \otimes \mathrm{B}$ measurable. $x \rightarrow F(t, x)$ is lower semicontinuous for almost every $t \in[0,1]$.

(5) There exists a function $f:[0,1] \times \Re^{+} \rightarrow \Re^{+}$such that $f(\cdot, s) \in$ $L^{1}\left(0,1 ; \Re^{+}\right)$for any $s \geq 0, f(t, \cdot)$ is continuous and increasing for almost every $t \in[0,1]$, and $|F(t, x)| \leq f(t,|x|)$ for almost every $t \in[0,1]$ and $x \in \Re$.

Assign to $F$ a multi-valued operator $\mathrm{F}: C[0,1] \rightarrow L^{1}[0,1]$ by letting $(2.1)$

$\mathrm{F}(y)=\left\{\omega \in L^{1}[0,1]: \omega(t) \in F(t, y(t)) ;\right.$ almost everywhere $\left.t \in[0,1]\right\}$.

Define the operator $S$ by

$$
S g(t)=h(t)+\int_{0}^{t} k(t, s) g(s) d s,
$$

where $g \in L^{1}[0,1]$, we can get that $S: L^{1}[0,1] \rightarrow C[0,1]$ is continuous.

Definition 2.1 [14]. Let $F:[0,1] \times \Re \rightarrow \Re$ be a multi-valued function with nonempty compact, convex values. We say $S \circ F$ is of supper semi-continuous type (upper semi-continuous type) if $S \circ \mathrm{F}$ is upper semi-continuous, completely continuous and has nonempty, compact, convex values.

Definition 2.2 [14]. Let $F:[0,1] \times \Re \rightarrow \Re$ be a multi-valued function with nonempty compact values. We say $F$ is of lower semi-continuous 
type (lower semi-continuous type) if $\mathrm{F}$ is lower semi-continuous and has nonempty, closed and decomposable values.

Theorem 2.3 [14]. Let $F:[0,1] \times \Re \rightarrow \Re$ be a multi-valued function with nonempty compact, convex values. Assume (1), (2), (3) and (5) are satisfied. Then $S \circ F$ is of supper semi-continuous type.

Theorem 2.4 [9]. Let $F:[0,1] \times \Re \rightarrow \Re$ be a multi-valued function with nonempty compact values. Assume (4) and (5) are satisfied. Then $F$ is of lower semi-continuous type.

Next we state a selection theorem [7] due to Bressan and Colombo. Let $Y$ be a metric space and $G: Y \rightarrow L^{1}[0,1]$ a multi-valued operator. We say $G$ has property (BC) if

(I) $G$ is lower semi-continuous.

(II) $G$ has nonempty closed and decomposable values.

Theorem $2.5[7]$. Let $Y$ be a separable metric space, and let $G: Y \rightarrow L^{1}[0,1]$ be a multi-valued operator which has property (BC). Then $G$ has a continuous selection, i.e., there exists a continuous function (single valued) $g: Y \rightarrow L^{1}[0,1]$ such that $g(y) \in G(y)$ for every $y \in Y$.

Lemma $2.6[\mathbf{2 1}]$. If $f:[0,1] \times \Re^{+} \rightarrow \Re^{+}$is continuous, and

$$
\limsup _{x \rightarrow+\infty} \frac{f(t, x)}{x} \leq R
$$

is uniformly for each $t \in[0,1]$, where $R \in(0,+\infty)$, then for each $r \in(0,+\infty)$, there exists a $w \in C^{1}[0,1]$ such that

$$
\left\{\begin{array}{l}
w^{\prime}(t) \geq f(t, w(t)), \\
w(t) \geq r
\end{array}\right.
$$

Finally we state three fixed point results which will be used in Section 3. 
Lemma 2.7 [20]. Let $S$ be a convex subset of a normed linear space $X$ and assume $0 \in S$. Let $F: S \rightarrow S$ be a continuous and compact map, and let the set $\{x \in S: x=\lambda F x$ for some $\lambda \in(0,1)\}$ be bounded. Then $F$ has at least one fixed point in $S$.

Theorem 2.8 [1, 22]. Let $C$ be a nonempty, closed, bounded, convex subset of a Banach space $E$. Then every compact, continuous map $F: C \rightarrow C$ has at least one fixed point.

Theorem $2.9[\mathbf{1 0}]$. Let $C$ be a closed, convex subset of a Banach space $E$ and $0 \in C$. Suppose $F: C \rightarrow C$ is an upper semi-continuous compact multi-valued map with nonempty, compact, convex values. Then there is an $x \in C$ such that $x \in F x$.

4. Solvability of Volterra integral inclusions. In this section, we apply the results obtained previously to study the solvability of the nonlinear Volterra integral inclusion (1.1).

Theorem 3.1. Let $F:[0,1] \times \Re \rightarrow \Re$ be a multi-valued function with nonempty, compact, convex values. Suppose (1), (2), (3) and (5) are satisfied. Then equation (1.1) has at least one solution in the space $C[0,1]$ provided that there exists an $m(t)$ with

$$
m(t) \geq a+K \int_{0}^{t} f(s, m(s)) d s, \quad t \in[0,1] .
$$

Proof. Define operator $N: C[0,1] \rightarrow C[0,1]$ by

$$
(N x)(t)=\left\{y(t) \in C[0,1]: y(t)=h(t)+\int_{0}^{t} k(t, s) v(s) d s ; v \in \mathrm{F}(x)\right\}
$$

Solving (1.1) is equivalent to the fixed point problem $x \in N x=$ $S \circ \mathrm{F}(x)$. Notice from Theorem 2.3 that $N$ is of upper semi-continuous type.

If we define $W=\{x \in C[0,1],|x(t)| \leq m(t)$, for all $t \in[0,1]\}$, then $W \subseteq C[0,1]$ is bounded and convex. 
Let $x \in W$, for each $y \in N x$. Then there exists $v \in \mathrm{F}(x)$ such that

$$
y(t)=h(t)+\int_{0}^{t} k(t, s) v(s) d s
$$

Then we have

$$
\begin{aligned}
|y(t)| & \leq|h(t)|+\left|\int_{0}^{t} k(t, s) v(s) d s\right| \\
& \leq a+K \int_{0}^{t} f(s, m(s)) d s \\
& \leq m(t),
\end{aligned}
$$

which implies that $N: W \rightarrow W$ is a bounded operator.

Applying Theorem 2.9 we may deduce the result immediately.

Next, we give existence results when $F$ is under the following conditions.

$\left(5^{\prime}\right)$ There exists a continuous function $f:[0,1] \times \Re^{+} \rightarrow \Re^{+}$, such that $f(t, \cdot)$ is increasing for each $t \in[0,1]$, and $|F(t, x)| \leq f(t,|x|)$ for almost every $t \in[0,1]$ and $x \in \Re$.

Theorem 3.2. Let $F:[0,1] \times \Re \rightarrow \Re$ be a multi-valued function with nonempty, compact, convex values. Suppose (1), (2), (3) and (5') are satisfied. Then there is at least one solution for (1.1) provided that there exists a constant $R>0$ such that

$$
\limsup _{x \rightarrow+\infty} \frac{f(t, x)}{x} \leq R
$$

is uniform for each $t \in[0,1]$.

Proof. By Lemma 2.6, we know there is a $m(t) \in C^{1}[0,1]$ such that

$$
\left\{\begin{array}{l}
m^{\prime}(t) \geq K f(t, m(t)) \\
m(t) \geq a
\end{array}\right.
$$


We can get

$$
m(t) \geq m(0)+K \int_{0}^{t} f(s, m(s)) d s \geq a+K \int_{0}^{t} f(s, m(s)) d s .
$$

From Theorem 3.1, we can obtain that the Volterra integral inclusion (1.1) has at least one continuous solution. Thus, the proof is complete.

At last we would like to discuss the Volterra integral inclusion (1.1) under the following conditions.

$\left(5^{\prime \prime}\right)$ There exist a function $l \in L^{1}\left(0,1 ; \Re^{+}\right)$and a nondecreasing continuous function $\Omega: \Re^{+} \rightarrow \Re^{+}$such that $|F(t, x)| \leq l(t) \Omega(|x|)$ for all $x \in \Re$ and almost every $t \in[0,1]$.

Theorem 3.3. Let $F:[0,1] \times \Re \rightarrow \Re$ be a multi-valued function with nonempty, compact, convex values. Suppose (1), (2), (3) and (5') are satisfied. Then there is at least one solution for (1.1) provided that

$$
\int_{0}^{1} l(s) d s<\int_{a}^{\infty} \frac{1}{K \Omega(s)} d s
$$

Proof. Define operator $G: S \rightarrow S$ by

$$
(G x)(t)=a+K \int_{0}^{t} l(s) \Omega(x(s)) d s, \quad t \in[0,1]
$$

for all $x \in S$, where $S=\{x(t) \geq 0, x(t) \in C[0,1]\}$. We know that $G$ is continuous and compact.

Next, we prove that the set $\{x: x=\lambda G x$ for some $\lambda \in(0,1)\}$ is bounded.

Let $x=\lambda G x$, i.e., for $t \in[0,1]$,

$$
x(t)=\lambda a+\lambda K \int_{0}^{t} l(s) \Omega(x(s)) d s .
$$


We have

$$
|x(t)| \leq a+K \int_{0}^{t} l(s) \Omega(|x(s)|) d s .
$$

Denoting by $u(t)$ the right-hand side of the above inequality, we know that $u(0)=a$ and $|x(t)| \leq u(t)$ for $t \in[0,1]$, and

$$
u^{\prime}(t)=K l(t) \Omega(|x(t)|) \leq K l(t) \Omega(u(t))
$$

for almost every $t \in[0,1]$.

This implies

$$
\int_{a}^{u(t)} \frac{1}{K \Omega(s)} d s \leq \int_{0}^{t} l(s) d s<\int_{a}^{\infty} \frac{1}{K \Omega(s)} d s
$$

for all $t \in[0,1]$.

This implies that there is a constant $r>0$ such that $u(t) \leq r$ for all $t \in[0,1]$. So, we get $|x(t)| \leq r$. By Lemma 2.7, we obtain that $G$ has at least one fixed point in $S$, i.e.,

$$
m(t)=a+K \int_{0}^{t} l(s) \Omega(m(s)) d s,
$$

where $m(t) \in S$.

From Theorem 3.1, the Volterra integral inclusion (1.1) has at least one continuous solution. Thus, the proof is complete.

Remark 3.4. In $[\mathbf{5}, \mathbf{1 7 - 1 9}]$ suppose $|F(t, x)| \leq a(t)+b(t)\|x\|$, where $a(\cdot), b(\cdot) \in L^{1}(0,1)$. In $[6]$ it is assumed that $\int_{a}^{\infty}(1 / \Omega(s)) d s=+\infty$. So, Theorems 3.1-3.3 improve and generalize the corresponding results $[\mathbf{5}, \mathbf{6}, \mathbf{1 7}-\mathbf{1 9}]$. Also, the result of Theorem 3.2 does not appear in $[\mathbf{5}$, 6, 13-15, 17-19].

Theorem $3.5[\mathbf{5}, \mathbf{1 7 - 1 9}]$. Under the assumptions of Theorem 3.3 and $|F(t, x)| \leq l(t)(|x|+1)$ for all $x \in \Re$ and almost every $t \in[0,1]$, then equation (1.1) has at least one solution in the space $C[0,1]$.

Proof. We know

$$
\int_{0}^{1} l(s) d s<+\infty=\int_{a}^{+\infty} \frac{1}{K(s+1)} d s .
$$


Therefore, by Theorem 3.3, we can obtain that equation (1.1) has at least one solution.

Theorem 3.6. Under the assumptions of Theorem 3.3, equation (1.1) has at least one solution in the space $C[0,1]$ provided that there exists a constant $R$ with

$$
a+K \int_{0}^{1} l(s) d s \Omega(R) \leq R .
$$

Proof. We can easily see that

$$
\int_{0}^{1} l(s) d s \leq \frac{R-a}{K \Omega(R)} \leq \int_{a}^{R} \frac{1}{K \Omega(s)} d s<\int_{a}^{+\infty} \frac{1}{K \Omega(s)} d s .
$$

Then, using Theorem 3.3, we can obtain that equation (1.1) has at least one solution in the space $C[0,1]$.

Remark 3.7. In [13-15], suppose there must exist an $R>0$, independent of $\lambda$, with $\|x\| \neq R$ for any solution to

$$
x=\lambda N x,
$$

for each $\lambda \in(0,1)$. It is difficult for us to find an $R$ satisfying the above conditions. Using the above theorems, we can easily obtain whether or not equation (1.1) has a solution. So we believe our theorems are better than the theorems of [13-15].

Finally, we discuss the nonlinear Volterra integral inclusion (1.1) where $F$ is not necessarily a convex value.

Theorem 3.8. Let $F:[0,1] \times \Re \rightarrow \Re$ be a multi-valued function with nonempty, compact values. Suppose (1), (2), (4) and (5) are satisfied. Then equation (1.1) has at least one solution in the space $C[0,1]$ provided that there exists an $m(t)$ with

$$
m(t) \geq a+K \int_{0}^{t} f(s, m(s)) d s, t \in[0,1] .
$$


Proof. Now Theorem 2.4 together with the Bressan Colombo selection theorem 2.5 imply that there exists a continuous function $g: C[0,1] \rightarrow$ $L^{1}[0,1]$ such that $g(x) \in \mathrm{F}(x)$ for all $x \in C[0,1]$.

Consider the problem

$$
x(t)=h(t)+\int_{0}^{t} k(t, s) g(x(s)) d s .
$$

It is obvious that if $x \in C[0,1]$ is a solution of problem (3.3), then $x$ is a solution to problem (1.1).

Define operator $N: C[0,1] \rightarrow C[0,1]$ by

$$
(N x)(t)=h(t)+\int_{0}^{t} k(t, s) g(x(s)) d s .
$$

We can show that $N$ is continuous and compact by the usual techniques.

We denote $W=\{x \in C[0,1],|x(t)| \leq m(t)$, for all $t \in[0,1]\}$. Then $W \subseteq C[0,1]$ is bounded and convex.

For each $x \in W$, we have

$$
\begin{aligned}
|(N x)(t)| & \leq|h(t)|+\left|\int_{0}^{t} k(t, s) g(x(s)) d s\right| \\
& \leq a+K \int_{0}^{t} f(s, m(s)) d s \\
& \leq m(t) .
\end{aligned}
$$

Then $N: W \rightarrow W$ is a bounded operator.

Apply Theorem 2.8 to deduce that (3.3) has a solution. Consequently, (1.1) has a solution.

Similarly to the proof of above theorems, we can obtain the following theorems.

Theorem 3.9. Let $F:[0,1] \times \Re \rightarrow \Re$ be a multi-valued function with nonempty, compact values. Suppose (1), (2), (4) and (5') are satisfied. Then there is at least one mild solution for (1.1) provided that there exists a constant $R>0$ such that

$$
\limsup _{x \rightarrow+\infty} \frac{f(t, x)}{x} \leq R
$$


is uniformly for each $t \in[0,1]$.

Theorem 3.10. Let $F:[0,1] \times \Re \rightarrow \Re$ be a multi-valued function with nonempty, compact values. Suppose (1), (2), (4) and (5") are satisfied. Then there is at least one mild solution for (1.1) provided that

$$
\int_{0}^{1} l(s) d s<\int_{a}^{\infty} \frac{1}{K \Omega(s)} d s .
$$

Theorem 3.11. Under the assumptions of Theorem 3.10 and $|F(t, x)| \leq l(t)(|x|+1)$ for all $x \in \Re$ and almost every $t \in[0,1]$, equation (1.1) has at least one solution in the space $C[0,1]$.

Theorem 3.12. Under the assumptions of Theorem 3.10, equation (1.1) has at least one solution in the space $C[0,1]$ provided that there exists a constant $R$ with

$$
a+K \int_{0}^{1} l(s) d s \Omega(R) \leq R .
$$

\section{REFERENCES}

1. R. Agarwal, M. Meehan and D. O'Regan, Fixed point theory and applications, Cambr. Tract. Math., Cambridge University Press, Cambridge, 2001.

2. R.P. Agarwal and D. O'Regan, Existence criteria for operator inclusions in abstract spaces, J. Comput. Appl. Math. 113 (2000), 183-193.

3. — Fixed-point theorems for countably condensing maps on Fréchet spaces, Comput. Math. Appl. 42 (2001), 909-916.

4. J.P. Aubin and A. Cellina, Differential inclusions, Springer-Verlag, Berlin, 1984.

5. E.P. Avgerinos, On the existence of solutions for Volterra integral inclusions in Banach spaces, J. Appl. Math. Stoch. Anal. 6 (1993), 261-270.

6. M. Benchohra, A note on integral inclusions in Banach spaces, Math. Pann. 11 (2000), 77-85.

7. A. Bressan and G. Colombo, Extensions and selections of maps with decomposable values, Stud. Math. 90 (1988), 70-85.

8. K. Deimling, Multivalued differential equations, Walter de Gruyter, Berlin, 1992. 
9. M. Frigon and A. Granas, Théorèmes d'existence pour des inclusions différentielles sans convexité, C.R. Acad. Sci. Paris 310 (1990), 819-822.

10. A. Granas and J. Dugundji, Fixed point theory, Springer-Verlag, New York, 2003.

11. S. Hu and N.S. Papageorgiou, Handbook of multivalued analysis, Vol. I: Theory, Kluwer Academic Publishers, Dordrecht, 1997.

12. M. Kisielewicz, Multivalued differential equations in separable Banach spaces, J. Optim. Theor. Appl. 37 (1998), 231-249.

13. D. O'Regan, Multivalued differential equations in Banach spaces, Comput. Math. Appl. 38 (1999), 109-116.

14. - Integral inclusions of upper semi-continuous or lower semi-continuous type, Proc. Amer. Math. Soc. 124 (1996), 2391-2399.

15. - Nonlinear alternatives for multivalued maps with applications to operator inclusions in abstract spaces, Proc. Amer. Math. Soc. 127 (1999), 3557-3564.

16. D. O'Regan and R. Precup, Fixed point theorems for set-valued maps and existence principles for integral inclusions, J. Math. Anal. Appl. 245 (2000), 594-612.

17. N.S. Papageorgiou, Volterra integral inclusions in Banach spaces, J. Integ. Equat. Appl. 1 (1988), 65-81.

18. - On nonconvex valued Volterra integral inclusions in Banach spaces, Czech. Math. 44 (1994), 631-648.

19. — On integral inclusions of Volterra type in Banach spaces, Czech. Math. 42 (1992), 693-714.

20. H. Schaefer, Über die methode der priori Schranhen, Math. Ann. 129 (1955), 415-416.

21. B. Shi, Theory and applications of differential equations, National Defense Industry Press, Beijing, China, 2005.

22. E. Zeidler, Nonlinear functional analysis and its applications (I) (Fixed point theorems), Springer, Berlin, 1992.

Department of Mathematics and Physics, Nanjing Institute of TechnolOGY, NANJing, 211100, P.R. China

Email address: zhutaoyzu@sina.cn

Department of Mathematics and Physics, Nanjing Institute of TechnolOGY, NANJing, 211100, P.R. China

Email address: csfunc@njit.edu.cn

Department of Mathematics, YangZhou University, YangZhou, 225002, P.R. CHINA

Email address: gli@yzu.edu.cn 